A Solution of the

Differential Equation of

Longitudinal Dispersion

in Porous Media

GEOLOGICAL SURVEY PROFESSIONAL PAPER 411-A 


\section{A Solution of the}

\section{Differential Equation of}

Longitudinal Dispersion

\section{in Porous Media}

By AKIO OGATA and R. B. BANKS

FLUID MOVEMENT IN EARTH MATERIALS

GEOLOGICAL SURVEY PROFESSIONAL PAPER 411-A

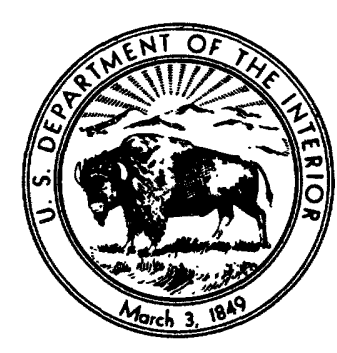

UNITED STATES GOVERNMENT PRINTING OFFICE, WASHINGTON : 1961 


\section{UNITED STATES DEPARTMENT OF THE INTERIOR}

STEWART L. UDALL, Secretary

GEOLOGICAL SURVEY

Thomas B. Nolan, Director

For sale by the Superintendent of Documents, U.S. Government Printing Office Washington 25, D.C. - Price 15 cents (paper cover) 


\section{CONTENTS}

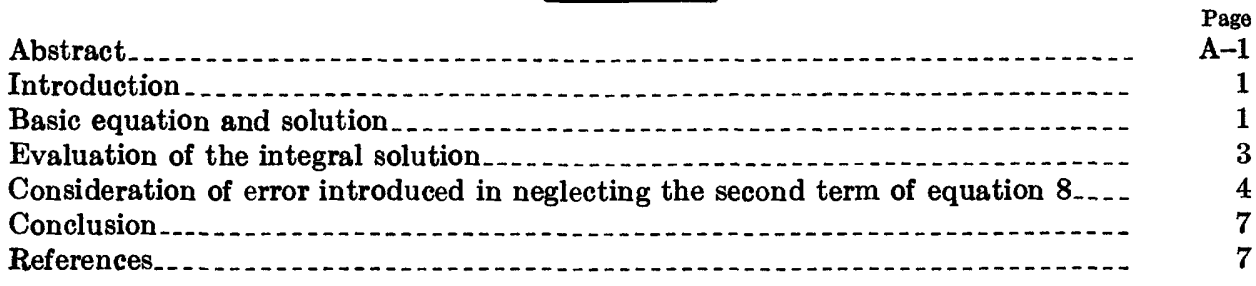

\section{ILLUSTRATIONS}

Figdre 1. Plot of equation 13

2. Comparison of theoretical and experimental results....

3. Plot of equation 18....

Page

$\mathbf{A}-\mathbf{5}$

5

III 
. 
FLUID MOVEMENT IN EARTH MATERIALS

\title{
A SOLUTION OF THE DIFFERENTIAL EQUATION OF LONGITUDINAL DISPERSION IN POROUS MEDIA
}

\author{
By Akio Ogata and R. B. Banks
}

\begin{abstract}
Published papers indicate that most investigators use the coordinate transformation $(x-u t)$ in order to solve the equation for dispersion of a moving fluid in porous media. Further, the boundary conditions $C=0$ at $x=\infty$ and $C=C_{0}$ at $x=-\infty$ for $t>0$ are used, which results in a symmetrical concentration distribution. This paper presents a solution of the differential equation that avoids this transformation, thus giving rise to an asymmetrical concentration distribution. It is then shown that this solution approaches that given by symmetrical boundary conditions, provided the dispersion coefficient $D$ is small and the region near the source is not considered.
\end{abstract}

\section{INTRODUCTION}

In recent years considerable interest and attention have been directed to dispersion phenomena in flow through porous media. Scheidegger (1954), deJong (1958), and Day (1956) have presented statistical means to establish the concentration distribution and the dispersion coefficient.

A more direct method is presented here for solving the differential equation governing the process of dispersion. It is assumed that the porous medium is homogeneous and isotropic and that no mass transfer occurs between the solid and liquid phases. It is assumed also that the solute transport, across any fixed plane, due to microscopic velocity variations in the flow tubes, may be quantitatively expressed as the product of a dispersion coefficient and the concentration gradient. The flow in the medium is assumed to be unidirectional and the average velocity is taken to be constant throughout the length of the flow field.

\section{BASIC EQUATION AND SOLUTION}

Because mass is conserved, the governing differential equation is determined to be

$$
\begin{aligned}
D \nabla^{2} C & =u \frac{\partial C}{\partial x}+\frac{\partial C}{\partial t} \\
\nabla^{2} & =\frac{\partial^{2}}{\partial x^{2}}+\frac{\partial^{2}}{\partial y^{2}}+\frac{\partial^{2}}{\partial z^{2}}
\end{aligned}
$$

where

$D=$ dispersion coefficient

$C=$ concentration of solute in the fluid

$u=$ average velocity of fluid or superficial velocity/ porosity of medium

$x=$ coordinate parallel to flow

$y, z=$ coordinates normal to flow

$t=$ time.

In the event that mass transfer takes place between the liquid and solid phases, the differential equation becomes

$$
D \nabla^{2} C=u \frac{\partial C}{\partial x}+\frac{\partial C}{\partial t}+\frac{\partial F}{\partial t}
$$

where $F$ is the concentration of the solute in the solid phase.

The specific problem considered is that of a semiinfinite medium having a plane source at $x=0$. Hence equation 1 becomes

$$
D \frac{\partial^{2} C}{\partial x^{2}}-u \frac{\partial C}{\partial x}=\frac{\partial C}{\partial t} .
$$

Initially, saturated flow of fluid of concentration, $C=0$, takes place in the medium. At $t=0$, the concentration of the plane source is instantaneously changed to $C=C_{0}$. Thus, the appropriate boundary conditions are

$$
\begin{aligned}
& C(0, t)=C_{0} ; t \geq 0 \\
& C(x, 0)=0 ; x \geq 0 \\
& C(\infty, t)=0 ; t \geq 0 .
\end{aligned}
$$

The problem then is to characterize the concentration as a function of $x$ and $t$.

To reduce equation 1 to a more familiar form, let

$$
C(x, t)=\Gamma(x, t) \exp \left(\frac{u x}{2 D}-\frac{u^{2} t}{4 D}\right)
$$


Substituting equation 4 into equation 1 gives

$$
\frac{\partial \Gamma}{\partial t}=D \frac{\partial^{2} \Gamma}{\partial x^{2}}
$$

The boundary conditions transform to

$$
\begin{aligned}
\Gamma(0, t) & =C_{0} \exp \left(u^{2} t / 4 D\right) ; t \geq 0 \\
\Gamma(x, 0) & =0 ; x \geq 0 \\
\Gamma(\infty, t) & =0 ; t \geq 0
\end{aligned}
$$

It is thus required that equation 5 be solved for a timedependent influx of fluid at $x=0$.

The solution of equation 5 may be obtained readily by use of Duhamel's theorem (Carslaw and Jaeger, 1947, p. 19):

If $C=F(x, y, z, t)$ is the solution of the diffusion equation for semi-infinite media in which the initial concentration is zero and its surface is maintained at concentration unity, then the solution of the problem in which the surface is maintained at temperature $\phi(t)$ is

$$
C=\int_{0}^{t} \phi(\lambda) \frac{\partial}{\partial t} F(x, y, z, t-\lambda) d \lambda .
$$

This theorem is used principally for heat conduction problems, but the above has been specialized to fit this specific case of interest.

Consider now the problem in which initial concentration is zero and the boundary is maintained at concentration unity. The boundary conditions are

$$
\begin{aligned}
& \Gamma(0, t)=1 ; t \geq 0 \\
& \Gamma(x, 0)=0 ; x \geq 0 \\
& \Gamma(\infty, t)=0 ; t \geq 0 .
\end{aligned}
$$

This problem is readily solved by application of the Laplace transform which is defined as

$$
\bar{\Gamma}(x, p)=\int_{0}^{\infty} e^{-p t} \Gamma(x, t) d t .
$$

Hence, if equation 5 is multiplied by $e^{-p t}$ and integrated term by term it is reduced to an ordinary differential equation

$$
\frac{d^{2} \bar{\Gamma}}{d x^{2}}=\frac{p}{D} \bar{\Gamma}
$$

The solution of equation 6 is

$$
\bar{\Gamma}=A e^{-q x}+B e^{q x}
$$

where

$$
q=\sqrt{p / D}
$$

The boundary condition as $x \rightarrow \infty$ requires that $B=0$ and boundary condition at $x=0$ requires that $A=1 / p$, thus the particular solution of the Laplace transformed equation is

$$
\bar{\Gamma}=\frac{1}{p} e^{-q x} .
$$

The inversion of the above function is given in any table of Laplace transforms (for example, Carslaw and Jaeger, p. 380). The result is

$$
\Gamma=1-\operatorname{erf}\left(\frac{x}{2 \sqrt{D t}}\right)=\frac{2}{\sqrt{\pi}} \int_{\frac{x}{2 \sqrt{D t}}}^{\infty} e^{-\eta^{2}} d \eta .
$$

Utilizing Duhamel's theorem, the solution of the problem with initial concentration zero and the timedependent surface condition at $x=0$ is

$$
\Gamma=\int_{0}^{t} \phi(\tau) \frac{\partial}{\partial t}\left[\frac{2}{\sqrt{\pi}} \int_{\frac{x}{2 \sqrt{D(t-\tau)}}}^{\infty} e^{-\eta^{2} d \eta}\right] d \tau
$$

Since $e^{-\eta^{2}}$ is a continuous function, it is possible to differentiate under the integral, which gives

$$
\frac{2}{\sqrt{\pi}} \frac{\partial}{\partial t} \int_{\frac{x}{2 \sqrt{D(t-\tau)}}}^{\infty} e^{-\eta^{2}} d \eta=\frac{x}{2 \sqrt{\pi D}(t-\tau)^{3 / 2}} e^{-x^{2} / 4 D(t-\tau)}
$$

Thus

$$
\Gamma=\frac{x}{\pi \sqrt{D}} \int_{0}^{t} \phi(\tau) e^{-x^{2} / 4 D(t-\tau)} \frac{d \tau}{(t-\tau)^{3 / 2}}
$$

Letting

$$
\lambda=x / 2 \sqrt{D(t-\tau)}
$$

the solution may be written

$$
\Gamma=\frac{2}{\sqrt{\pi}} \int_{\frac{x}{2 \sqrt{D} t}}^{\infty} \phi\left(t-\frac{x^{2}}{4 D \lambda^{2}}\right) e^{-\lambda^{2}} d \lambda
$$

Since $\phi(t)=C_{\mathrm{o}} \exp \left(u^{2} t / 4 D\right)$ the particular solution of the problem may be written,

$$
\begin{aligned}
\Gamma(x, t)=C_{0} \frac{2}{\sqrt{\pi}} e^{\frac{u^{2} t}{\mathcal{D} D}}\left\{\int_{0}^{\infty} \exp \left(-\lambda^{2}-\frac{\epsilon^{2}}{\lambda^{2}}\right) d \lambda\right. \\
\\
\left.\quad-\int_{0}^{\alpha} \exp \left(-\lambda^{2}-\frac{\epsilon^{2}}{\lambda^{2}}\right) d \lambda\right\}
\end{aligned}
$$

where $\epsilon=\frac{u x}{4 D^{2}}$ and $\alpha=\frac{x}{2 \sqrt{D t}}$. 
DIFFERENTIAL EQUATION OF LONGITUDINAL DISPERSION IN POROUS MEDIA

A-3

EVALUATION OF THE INTEGRAL SOLUTION

The integration of the first term of equation 9 gives

$$
\int_{0}^{\infty} e^{-\lambda^{2}-e^{2}} d \lambda=\frac{\sqrt{\pi}}{2} e^{-2 \epsilon}
$$

(Pierce, 1956, p. 68)

For convenience the second integral may be expressed in terms of error function (Horenstein, 1945), because this function is well tabulated.

Noting that

$$
\begin{aligned}
-\lambda^{2}-\frac{\epsilon^{2}}{\lambda^{2}} & =-\left(\lambda+\frac{\epsilon}{\lambda}\right)^{2}+2 \epsilon \\
& =-\left(\lambda-\frac{\epsilon}{\lambda}\right)^{2}-2 \epsilon
\end{aligned}
$$

The second integral of equation 9 may be written

$$
\begin{gathered}
I=\int_{0}^{\alpha} \exp \left[-\lambda^{2}-\frac{\epsilon^{2}}{\lambda^{2}}\right] d \lambda=\frac{1}{2}\left\{e^{2 \epsilon} \int_{0}^{\alpha} \exp \left[-\left(\lambda+\frac{\epsilon}{\lambda}\right)^{2}\right] d \lambda\right. \\
\left.+e^{-2 \epsilon} \int_{0}^{\alpha} \exp \left[-\left(\lambda-\frac{\epsilon}{\lambda}\right)^{2}\right] d \lambda\right\}
\end{gathered}
$$

Since the method of reducing integral to a tabulated function is the same for both integrals in the right side of equation 10, only the first term is considered. Let $z=\epsilon / \lambda$ and adding and subtracting

$$
e^{2 \epsilon} \int_{\epsilon / \alpha}^{\infty} \exp \left[-\left(\frac{\epsilon}{z}+z\right)^{2}\right] d z
$$

the integral may be expressed

$$
\begin{aligned}
I_{1}=e^{2 \epsilon} \int_{0}^{\alpha} \exp \left[-\left(\lambda+\frac{\epsilon}{\lambda}\right)^{2}\right] d \lambda & \\
=-e^{2 \epsilon} \int_{\epsilon / \alpha}^{\infty}\left(1-\frac{\epsilon}{z^{2}}\right) & \exp \left[-\left(\frac{\epsilon}{z}+z\right)^{2}\right] d z \\
& +e^{2 \epsilon} \int_{\epsilon / \alpha}^{\infty} \exp \left[-\left(\frac{\epsilon}{z}+z\right)^{2}\right] d z .
\end{aligned}
$$

Further, let

$$
\beta=\left(\frac{\epsilon}{z}+z\right)
$$

in the first term of the above equation, then

$$
I_{1}=-e^{2 \epsilon} \int_{\alpha+\frac{\epsilon}{\alpha}}^{\infty} e^{-\beta^{2}} d \beta+e^{2 \epsilon} \int_{\epsilon / \alpha}^{\infty} \exp \left[-(\epsilon+z)^{2}\right] d z .
$$

Similar evaluation of the second integral of equation 10 gives

$$
\begin{aligned}
I_{2}=e^{-2 \epsilon} \int_{\epsilon / \alpha}^{\infty} \exp \left[-\left(\frac{\epsilon}{z}-z\right)^{2}\right] d z & \\
& -e^{-2 \epsilon} \int_{\epsilon / \alpha}^{\infty} \exp \left[-\left(\frac{\epsilon}{z}-z\right)^{2}\right] d z .
\end{aligned}
$$

Again substituting $-\beta=\frac{\varepsilon}{2}-z$ into the first term, the result is

$$
I_{2}=e^{-2 \epsilon} \int_{\frac{\epsilon}{\alpha}-\alpha}^{\infty} e^{-\beta^{2}} d \beta-e^{-2 \epsilon} \int_{\epsilon / \alpha}^{\infty} \exp \left[-\left(\frac{\epsilon}{z}-z\right)^{2}\right] d z .
$$

Noting that

$$
\int_{\epsilon / \alpha}^{\infty} \exp \left[-\left(z+\frac{\epsilon}{z}\right)^{2}+2 \epsilon\right] d z=\int_{\epsilon / \alpha}^{\infty} \exp \left[-\left(\frac{\epsilon}{z}-z\right)^{2}-2 \epsilon\right] d z
$$

substitution into equation 10 gives

$$
I=e^{-2 \epsilon} \int_{\frac{\epsilon}{\alpha}-\alpha}^{\infty} e^{-\beta^{2}} d \beta-e^{2 \epsilon} \int_{\frac{\epsilon}{\alpha}+\alpha}^{\infty} e^{-\beta^{2}} d \beta .
$$

Thus, equation 9 may be expressed

$$
\begin{aligned}
\Gamma(x, t)=\frac{2 C_{0}}{\sqrt{\pi}} e^{\frac{u^{2} t}{4 D}}\left\{\frac{\sqrt{\pi}}{2} e^{-2 \epsilon}-\right. \\
\left.\frac{1}{2}\left[e^{-2 \epsilon} \int_{\frac{\epsilon}{\alpha}-\alpha}^{\infty} e^{-\beta^{2}} d \beta-e^{2 \epsilon} \int_{\frac{\epsilon}{\alpha}+\alpha}^{\infty} e^{-\beta^{2}} d \beta\right]\right\}
\end{aligned}
$$

However, by definition,

$$
e^{2 \epsilon} \int_{\alpha+\frac{\epsilon}{\alpha}}^{\infty} e^{-\beta^{2}} d \beta=\frac{\sqrt{\pi}}{2} e^{2 \epsilon} \operatorname{erfc}\left(\alpha+\frac{\epsilon}{\alpha}\right)
$$

also,

$$
e^{-2 \epsilon} \int_{\frac{\epsilon}{\alpha}-\alpha}^{\infty} e^{-\beta^{2}} d \beta=\frac{\sqrt{\pi}}{2} e^{-2 \epsilon}\left[1+\operatorname{erf}\left(\alpha-\frac{\epsilon}{\alpha}\right)\right] .
$$

Writing equation 11 in terms of the error functions

$$
\Gamma(x, t)=\frac{C_{0}}{2} e^{\frac{u^{2} t}{4 D}}\left[e^{2 \epsilon} \operatorname{erfc}\left(\alpha+\frac{\epsilon}{\alpha}\right)+e^{-2 \epsilon} \operatorname{erfc}\left(\alpha-\frac{\epsilon}{\alpha}\right)\right] .
$$

Thus, substituting into equation 4 the solution is

$$
\frac{C}{C_{0}}=\frac{1}{2}\left[\operatorname{erfc}\left(\alpha-\frac{\epsilon}{\alpha}\right)+e^{4 \epsilon} \operatorname{erfc}\left(\alpha+\frac{\epsilon}{\alpha}\right)\right] .
$$


Resubstituting for $\epsilon$ and $\alpha$ gives

$$
\frac{C}{C_{0}}=\frac{1}{2}\left\{\operatorname{erfc}\left(\frac{x-u t}{2 \sqrt{D t}}\right)+e^{\frac{u x}{D}} \operatorname{erfc}\left(\frac{x+u t}{2 \sqrt{D t}}\right)\right\}
$$

which may be written in terms of dimensionless parameters,

$$
\frac{C}{C_{0}}=\frac{1}{2}\left[\operatorname{erfc}\left(\frac{1-\xi}{2 \sqrt{\xi \eta}}\right)+\exp \left(\frac{1}{\eta}\right) \operatorname{erfc}\left(\frac{1+\xi}{2 \sqrt{\xi \eta}}\right)\right]
$$

where $\xi=u t / x$ and $\eta=D / u x$.

Where boundaries are symmetrical the solution of the problem is given by the first term of equation 13 . This symmetrical system was considered by Dankwerts (1953) and Day (1956), utilizing different analytical methods. The second term in equation 13 is thus due to the asymmetric boundary imposed in the more general problem. However, it should be noted also that if a point a great distance away from the source is considered, then it is possible to approximate the boundary condition by $C(-\infty, t)=C_{0}$, which leads to a symmetrical solution.

A plot on logarithmic probability graph of the above solution is given in figure 1 for various values of the dimensionless group $\eta=D / u x$. The figure shows that as $\eta$ becomes small the concentration distribution becomes nearly symmetrical about the value $\xi=1$. However, for large values of $\eta$ asymmetrical concentration distributions become noticeable. This indicates that for large values of $D$ or small values of distance $x$ the contribution of the second term in equation 13 becomes significant as $\xi$ approaches unity.

Experimental results present further evidence (for example, Orlob, 1958; Ogata, Dispersion in Porous Media, doctoral dissertation, Northwestern Univ., 1958) that the distribution is symmetrical for values of $x$ chosen some distance from the source. An example of experimental break-through curves obtained for dispersion in a cylindrical vertical column is shown as figure 2. The theoretical curve was obtained by neglecting the second term of equation 13.

\section{CONSIDERATION OF ERROR INTRODUCED IN NEGLECTING THE SECOND TERM OF EQUATION 8}

Experimental data obtained give strong indication that in the region of flow that is of particular interest it is necessary to consider only the first term of equation 13. Owing to complexity of the overall problem of determining the error, it would facilitate analysis to determine the value of $\xi$ at which the function $e^{1 / \eta}$ erfc $\left(\frac{1+\xi}{2 \sqrt{\xi \eta}}\right)$ is a maximum. This then will enable the determination of the value of $\eta$ at which equation 13 may be reduced to

$$
\frac{C}{C_{0}}=\frac{1}{2} \operatorname{erfc}\left(\frac{1-\xi}{2 \sqrt{\xi \eta}}\right)
$$

without introducing errors in excess of experimental errors.

The necessary condition that the function $f(\eta, \xi)$ is a stationary point is given by

$$
\frac{\partial f}{\partial \xi}=0=\frac{\partial f}{\partial \eta}
$$

To determine whether the function is either maximum or minimum at a given point the sufficient conditions are given by

(a) $\left(\frac{\partial^{2} f}{\partial \xi^{2}}\right)\left(\frac{\partial^{2} f}{\partial \eta^{2}}\right)-\left(\frac{\partial^{2} f}{\partial \xi \partial \eta}\right)^{2}>0$

(b) Maxima, $\frac{\partial^{2} f}{\partial \xi^{2}}<0$, consequently $\frac{\partial^{2} f}{\partial \eta^{2}}<0$

(c) Minima, $\frac{\partial^{2} f}{\partial \xi^{2}}>0$, consequently $\frac{\partial^{2} f}{\partial \eta^{2}}>0$

(Irving and Mullineux, 1959, pp. 183-187)

Further, if 16(a) is greater than zero, the stationary point is called a saddle point.

Let

$$
f(\xi, \eta)=e^{1 / \eta} \operatorname{erfc}\left(\frac{1+\xi}{2 \sqrt{\xi \eta}}\right)
$$

Differentiating the function

$$
\frac{\partial f}{\partial \xi}=-\frac{1}{2 \sqrt{\pi \eta}}\left[(\xi-1) \xi^{-3 / 2}\right] e^{-\epsilon^{2}}
$$

where $\epsilon^{2}=(1-\xi)^{2} / 4 \xi \eta$. From the above expression it can be seen that $\xi=1$ and $\xi=\infty$ are the stationary points of the function.

The second differentials can readily be obtained by direct methods. The results are

$$
\frac{\partial^{2} f}{\partial \xi^{2}}=-\frac{1}{2 \sqrt{\pi \eta}} e^{-\epsilon^{2}}\left[\frac{1}{2}(3-\xi) \xi^{-5 / 2}+(\xi-1)^{2}(1+\xi) \xi^{-7 / 2}\right]
$$

and

$$
\begin{aligned}
\frac{\partial^{2} f}{\partial \eta^{2}}=\left(\eta^{-3}+\frac{1}{2} \eta^{-4}\right) e^{1 / \eta} \operatorname{erfc} & \left(\frac{1+\xi}{2 \sqrt{\xi \eta}}\right) \\
+ & \frac{1}{2 \sqrt{\pi \xi}}\left[\frac{1}{4}(1-\xi)^{2} \eta^{-7 / 2}-\frac{3}{2} \eta^{-5 / 2}\right]
\end{aligned}
$$

also

$$
\frac{\partial^{2} f}{\partial \xi \partial \eta}=\frac{1}{2 \sqrt{\pi}}(\xi-1) \xi^{-3 / 2} \eta^{-3 / 2} e^{-\epsilon^{2}}\left[\frac{1}{2}-\frac{\epsilon^{2}}{4}\right] .
$$


DIFFERENTIAL EQUATION OF LONGITUDINAL DISPERSION IN POROUS MEDIA

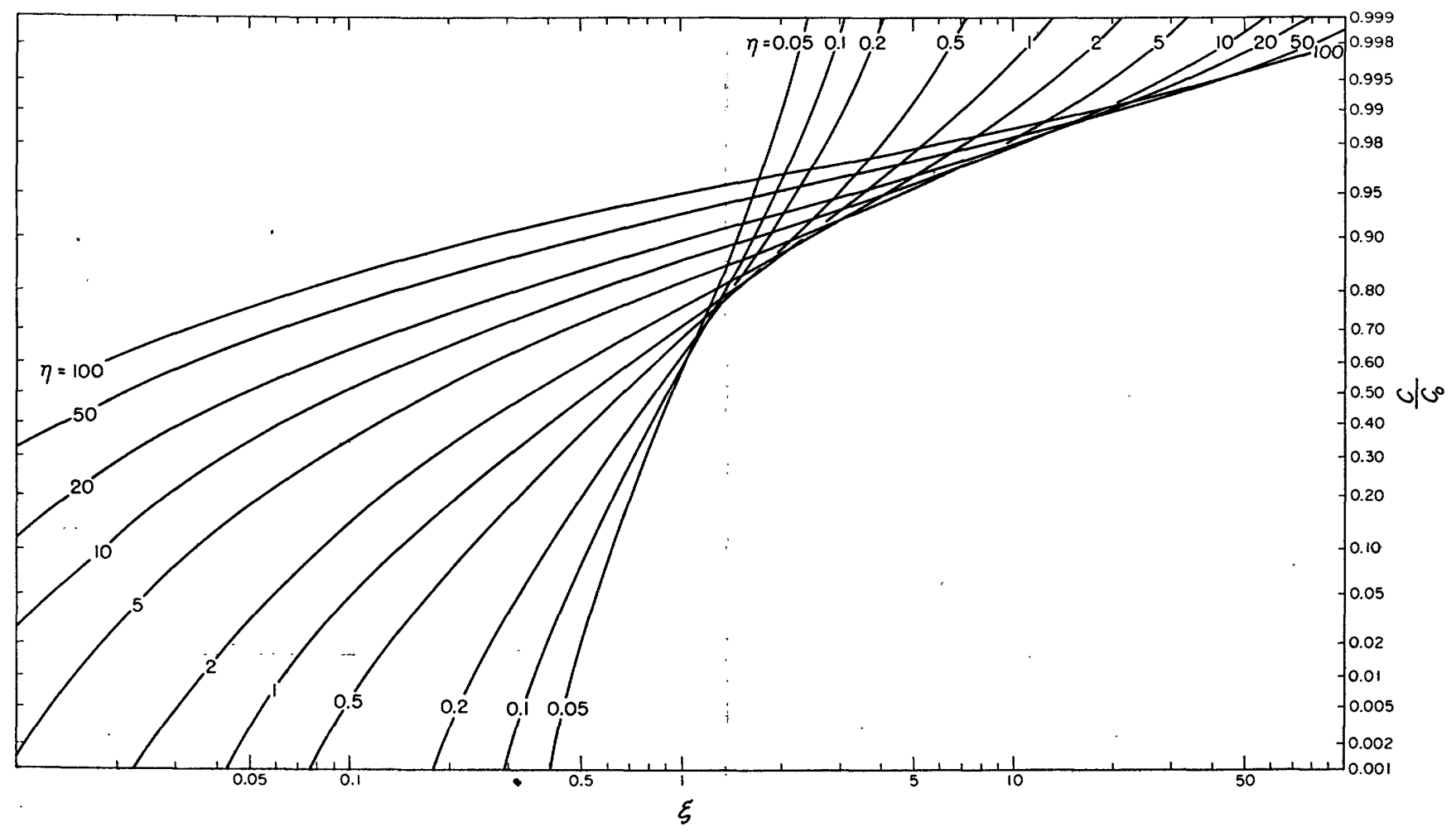

FIOURE 1.-Plot of equation 13.

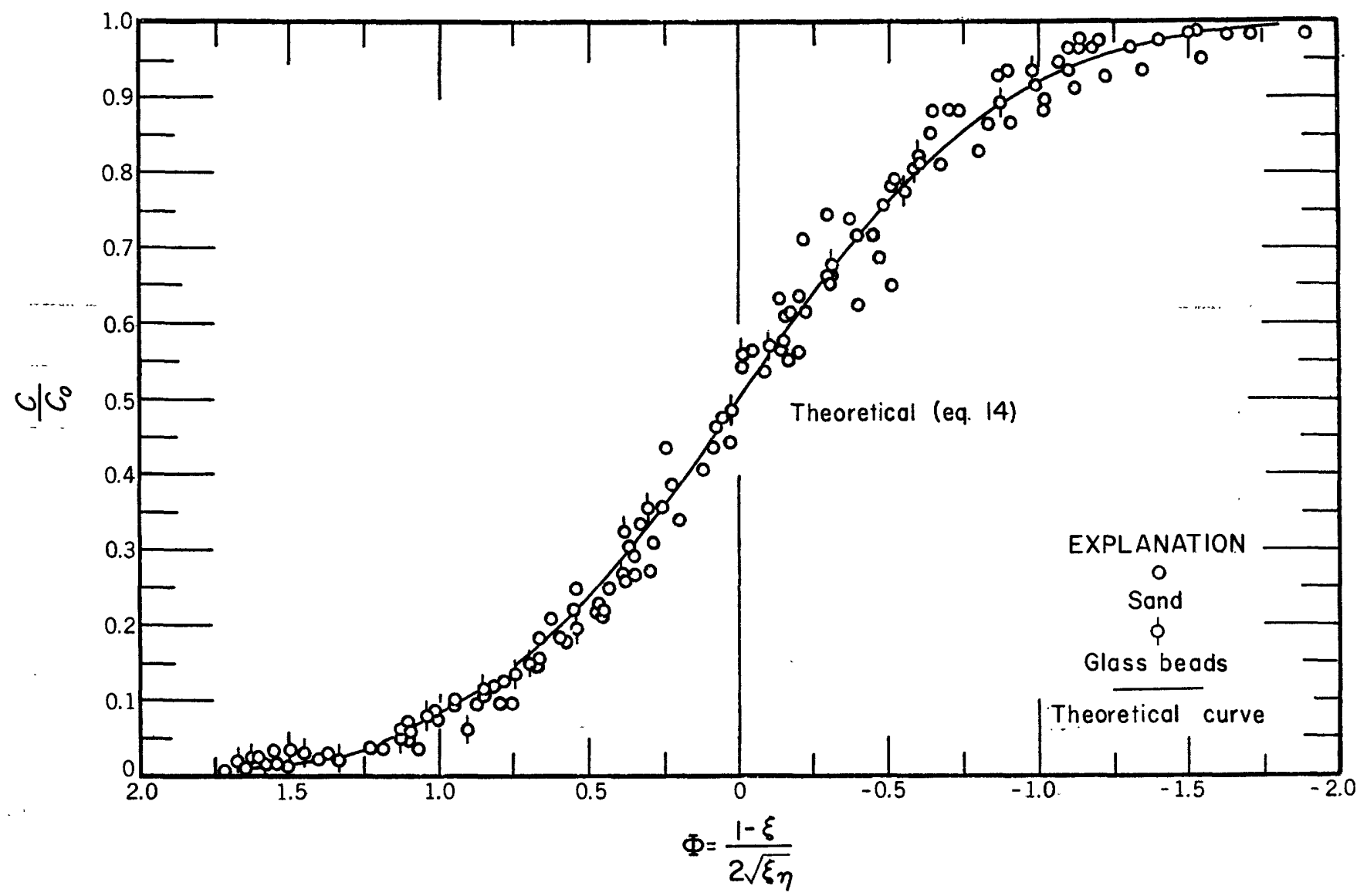

FIOURE 2.-Comparison of theoretical and experimental results. 
At point $\xi=1$, the following expressions are obtained:

$$
\begin{aligned}
& \left.\frac{\partial^{2} f}{\partial \xi^{2}}\right|_{\xi=1}=-(4 \pi \eta)^{-1 / 2} \\
& \left.\frac{\partial^{2} f}{\partial \eta^{2}}\right|_{\xi=1}=\left(\eta^{-3}+\frac{1}{2} \eta^{-4}\right) e^{1 / \eta} \operatorname{erfc}\left(\eta^{-1 / 2}\right)-\frac{3}{4 \sqrt{\pi}} \eta^{-5 / 2} \\
& \left.\frac{\partial^{2} f}{\partial \xi \partial \eta}\right|_{\xi=1}=0
\end{aligned}
$$

It can be shown that

$$
\left.\frac{\partial^{2} f}{\partial \eta^{2}}\right|_{\xi=1}<0
$$

for all values of $\eta$ by numerical consideration of the expansion of the complementary error function.

Accordingly, the function $e^{1 / \eta} \operatorname{erfc}\left(\frac{1+\xi}{2 \sqrt{\xi \eta}}\right)$ satisfies conditions 16 (a) and $16(\mathrm{~b})$, indicating that maxima of the function occurs at $\xi=1$. The point at infinity is not considered here since both terms of equation 13 approach zero as $\xi$ approaches infinity, which is indicative of a minimum condition.
There are no general analytical means available by which it is possible to obtain a general expression to determine the error involved in neglecting the second term of equation 13. Accordingly, consideration will be given in obtaining a reasonable numerical value of $\eta$ for which the second term in equation 13 may be neglected. Consider equations 13 and 14 at $\xi=1$. Note that equation 14 reduces the value of $\frac{C}{C_{0}}=\frac{1}{2}$, while
equation 13 gives

$$
\frac{C}{C_{0}}=\frac{1}{2}\left[1+e^{\lambda^{2}} \operatorname{erfc} \lambda\right] \text { at } \xi=1
$$

where $\lambda=\frac{1}{\sqrt{\eta}}$. The function $e^{\lambda^{2}}$ erfe $\lambda$ is tabulated in Carslaw and Jaeger (1948) up to the value $\lambda=3.0$. For large values of $\lambda$ or small values of $\eta$ the function may be approximated by

$$
e^{\lambda^{2}} \operatorname{erfc} \lambda=\frac{1}{\sqrt{\pi}}\left[\frac{1}{\lambda}-\frac{1}{2 \lambda^{3}}+\frac{1,3}{2^{2} \lambda^{5}}-\cdots\right]
$$

Hence, equation 18 may be readily computed.

A semilogarithmic plot of equation 18 is given in

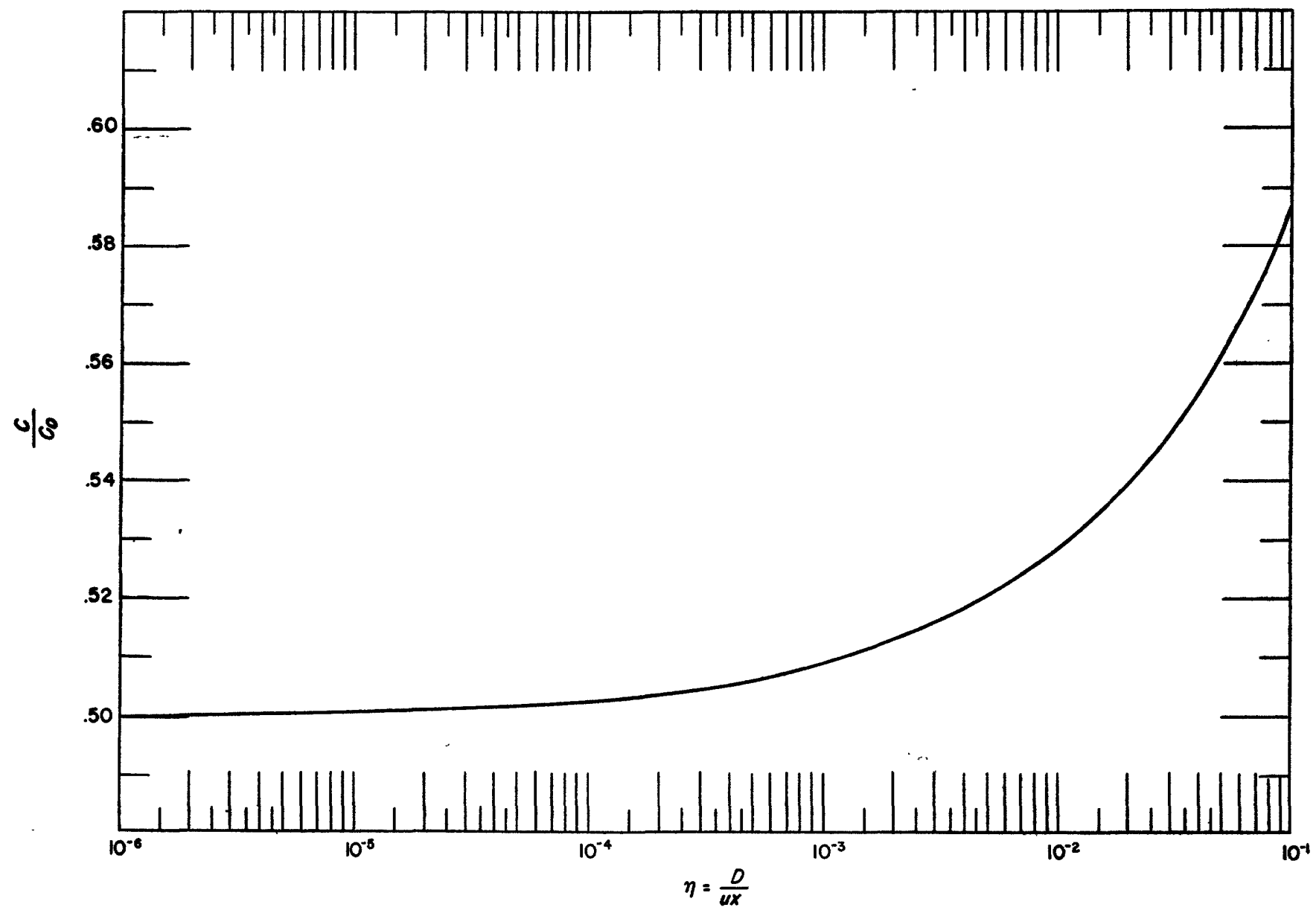

Fiaure 3.-Plot of equation 18. 
figure 3. It indicates that for values of $\eta<0.002 \mathrm{a}$ maximum error of less than 3 percent is introduced by neglecting the second term of equation 13.

In all experiments reported, the dispersion coefficient $D$ ranged from approximately $10^{-2}$ to $10^{-4} \mathrm{~cm}^{2} / \mathrm{sec}$. Further, it has been established that $D$ is proportional to the velocity, hence the relationship $D=D_{m} u$ may be written, where $D_{m}$ is the proportionality constant which is believed to be dependent on the media. Accordingly, since $\eta=D / u x, \eta$ may be expressed as $\eta=D_{m / x}$. This then indicates that for $x>5 D_{m} \times 10^{2}$ the second term of equation 13 becomes negligible. Orlob and Radhakrishna (1958) obtained values of $D_{m}$ ranging from 0.09 $\mathrm{cm}$ to $2.79 \mathrm{~cm}$. Using these values, measurements must be obtained at values of $x$ greater than $45 \mathrm{~cm}$ or 1395 $\mathrm{cm}$. However, if an error of 5 percent is permitted, the above values are reduced by a factor of 4 , thus $x$ must be greater than $10 \mathrm{~cm}$ or $350 \mathrm{~cm}$.

\section{CONCLUSION}

Consideration of the governing differential equation for dispersion in flow through porous media gives rise to a solution that is not symmetrical about $x=u t$ for large values of $\eta$. Experimental evidence, however, reveals that $D$ is small. This indicates that, unless the region close to the source is considered, the concentra- tion distribution is approximately symmetrical. Theoretically, $\frac{C}{C_{0}} \rightarrow \frac{1}{2}$ only as $\eta \rightarrow 0$; however, only errors of the order of magnitude of experimental errors are introduced in the ordinary experiments if a symmetrical solution is assumed instead of the actual asymmetrical one.

\section{REFERENCES}

Carslaw, H. S., and Jaeger, J. C., 1947, Conduction of heat in solids: Oxford Univ. Press, 386 p.

Dankwerts, P. V., 1953, Continuous flow system distribution of residence times: Chem. Eng. Sci., v. 2, p. 1-13.

Day, P. R., 1956, Dispersion of a moving salt-water boundary advancing through saturated sand: Am. Geophys. Union Trans., v. 37, p. 595-601.

deJong, G. de J., 1958, Longitudinal and transverse diffusion in granular deposits: Am. Geophys. Union Trans., v. 39, p. 67-74.

Horenstein, W., 1945, On certain integrals in the theory of heat conduction: Appl. Math. Quart., v. 3, p. 183-187.

Irving, J., and Mullineux, N., 1959, Mathematics in physics and engineering: Academic Press, 883 p.

Orlob, G. T., and Radhakrishna, G. N., 1958, The effects of entrapped gases on the hydraulic characteristics of porous media: Am. Geophys. Union Trans., v. 39, p. 648-659.

Pierce, B. O., and Foster, R. M., 1956, A short table of integrals: Boston, Mass., Ginn and Co., 189 p.

Scheidegger, A. E., 1954, Statistical hydrodynamics in porous media: Appl. Phys. Jour., v. 25, p. 994-1001. 

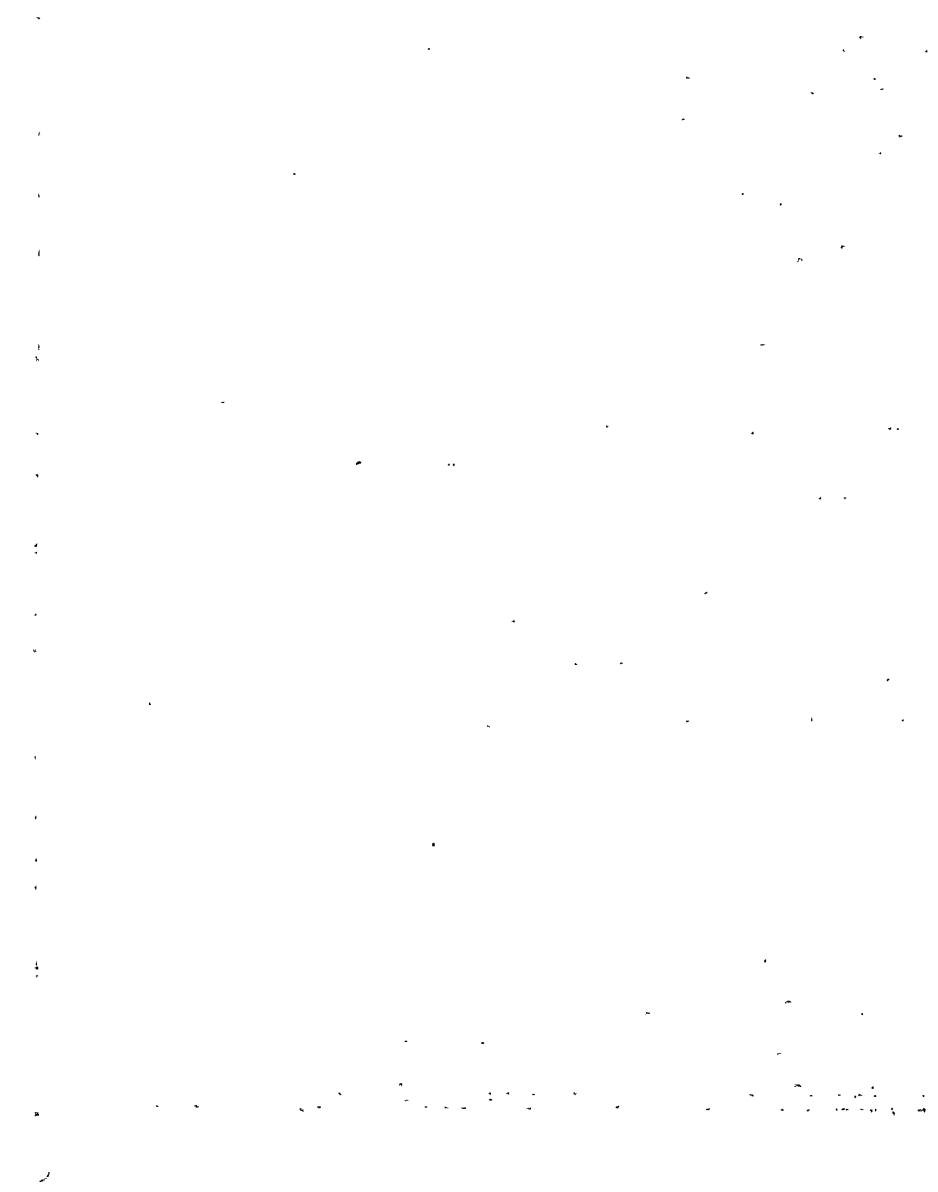